ApJ LetTers, IN PRESS

Preprint typeset using $\mathrm{AT}_{\mathrm{E} X} \mathrm{X}$ style emulateapj v. 11/10/09

\title{
A HYPOTHESIS FOR THE COLOR DIVERSITY OF THE KUIPER BELT
}

\author{
M.E. BROWN \\ Division of Geological and Planetary Sciences, California Institute of Technology, Pasadena, CA 91125 \\ E.L. SCHALLER \\ NASA Dryden Aircraft Operations Facility, Palmdale, CA 93550 and National Suborbital Education and Research Center, University of \\ North Dakota, Grand Forks, ND, 85202 \\ W.C. Fraser \\ Division of Geological and Planetary Sciences, California Institute of Technology, Pasadena, CA 91125 \\ ApJ Letters, in press
}

\begin{abstract}
We propose a chemical and dynamical process to explain the surface colors of the Kuiper belt. In our hypothesis, the initial bulk compositions of the bodies themselves can be quite diverse - as is seen in comets - but the early surface compositions are set by volatile evaporation after the objects are formed. Strong gradients in surface composition, coupled with UV and particle irradiation, lead to the surface colors that are seen today. The objects formed in the inner part of the primordial belt retain only $\mathrm{H}_{2} \mathrm{O}$ and $\mathrm{CO}_{2}$ as the major ice species on their surfaces. Irradiation of these species plausibly results in the dark neutrally colored centaurs and KBOs. Object formed further in the disk retain $\mathrm{CH}_{3} \mathrm{OH}$, which has been shown to lead to brighter redder surfaces after irradiation, as seen in the brighter redder centaurs and KBOs. Objects formed at the current location of the cold classical Kuiper belt uniquely retain $\mathrm{NH}_{3}$, which has been shown to affect irradiation chemistry and could plausibly lead to the unique colors of these objects. We propose observational and experimental tests of this hypothesis.

Subject headings: solar system: Kuiper belt — solar system: formation — astrochemistry
\end{abstract}

\section{INTRODUCTION}

Nearly twenty years after the realization that the region beyond Neptune is a depository for vast numbers of objects, one of the first discoveries about the physical properties of these Kuiper belt objects (KBOs) - that they span a wider range of colors than most other solar system populations - remains unexplained (Jewitt \& Luu 1998; Luu \& Jewitt 1996; Doressoundiram et al. 2008). The surfaces of KBOs range from those which are neutrally reflecting - and thus appear to have essentially solar colors - to some of the reddest objects known in the solar system. The full range of colors is mixed at what appears to be nearly random throughout the outer solar system (Morbidelli \& Brown 2005). Early hypotheses of randomized collisional excavation (Luu \& Jewitt 1996) or velocity dependent impact resurfacing (Stern 2002) have proven incapable of reproducing the features of the observations (Doressoundiram et al.|2008), yet no alternatives have been proposed.

We propose a chemically and dynamically plausible hypothesis where the surface compositions and thus colors of KBOs and their progeny are set by formation-location dependent volatile loss in the early solar system. In the next section we present the observational constraints on KBO colors. Next we detail the volatile-loss gradient hypothesis, and finally, we present predictions for this hypothesis.

mbrown@caltech.edu

\section{OBSERVATIONAL CONSTRAINTS}

While the causes of color variation among KBOs remain unclear, key observational constraints provide important clues for understanding the surfaces of these objects.

Cold classical KBOS. While most of the Kuiper belt appears to be composed of essentially the same mixture of gray to red objects (Morbidelli \& Brown 2005; Doressoundiram et al. 2008) one dynamical region stands out for its homogeneous composition. The cold classical Kuiper belt was first identified as a dynamically unique region of the Kuiper belt - a difficult-to-explain overabundance of low inclination, dynamically cold objects beyond about 41 AU (Brown 2001). Subsequent observations revealed that these objects shared a common red coloring (Trujillo \& Brown 2002). These cold classical KBOs are now also know to be unique in their lack of large bodies (Levison \& Stern 2001), their higher abundance of satellites (Noll et al. 2008), and their different size distribution (Fraser et al. 2010). Preliminary results also suggest that the cold classical KBOs have higher albedos than those of the remaining population (Brucker et al. 2009). All of these properties appear to signify a population with a different - and perhaps unique - formation location or history. Explaining the uniformly red color of these objects is critical to any understanding of Kuiper belt surface colors.

Centaurs. Centaurs - former KBOs currently occupying short-lived giant planet-crossing orbits - provide a second important constraint on KBO colors. Centaurs 
are derived from the Kuiper belt and should start with the surface chemistry range inherent in that population.

While the range of centaur colors generally covers the full range of colors covered by the Kuiper belt, the centaurs appear deficient in colors in the middle part of the range, giving the distribution of centaur colors a bimodal appearance (Tegler et al. 2008). This bimodality has been hypothesized to be due to a surface modification as centaurs are heated as they approach closer to perihelion. None of the specific models proposed, however, is able to fit the detailed spectral, albedo, and color observations (Schaller et al. 2009).

Local conditions. The colors of scattered, resonant, and hot classical Kuiper belt objects (that is, everything except cold classical Kuiper belt objects and centaurs) are uncorrelated with any current dynamical parameter. This lack of correlation, particularly with semi-major axis or perihelion/aphelion distance argues strongly that local heating, UV irradiation, and solar wind and cosmic ray bombardment (Cooper et al. 2003) are not responsible for the varying colors of the Kuiper belt. Local conditions appear to have no primary influence on the colors of KBOs.

Binary KBOS. Careful measurement of the colors of the separate components of binary KBOs has shown a tight correlation over the full range of Kuiper belt colors (Benecchi et al. 2009). The colors of two KBOs in orbit around each other are almost always nearly identical. This fact immediately rules out stochastic processes such as collisions for the causes of these Kuiper belt colors. Indeed, given the lack of correlation of color with local conditions, the nearly identical colors of binary KBOs argues that colors are simply primordial. If binary KBOs were formed by early mutual capture in a quiescent disk (Goldreich et al. 2002), the two component would likely have formed in very similar locations. If, alternatively, binary KBOs were formed in an initial gravitational collapse (Nesvorný et al. 2010), the objects would of necessity have formed at the same location and of the same materials.

Albedos. The albedos of KBOs are an important indicator of surface compositions. While measuring albedos of distant KBOs is difficult and reliable results are known for mainly the largest (and thus perhaps most unrepresentative) objects, closer, warmer centaurs have better characterized albedos. One result that appears robust is that the optically red centaurs have higher albedos than the optically blue centaurs, with the red centaur albedos clustering around $4 \%$ and the blue centaur albedos a much higher 11\% (Stansberry et al. 2006).

\section{THE VOLATILE-LOSS GRADIENT HYPOTHESIS}

If current Kuiper belt colors were set primarily by their formation location, as the similar colors of binary components and lack of correlation of color with current environment suggests, the fact that KBOs of all colors are now present throughout the Kuiper belt strongly argues that substantial mixing has occurred after formation. Instability models such as the Nice model (Tsiganis et al. 2005; Levison et al. 2008) provide natural mechanisms for this mixing, as objects which formed in the closer and more massive primordial disk and dispersed essentially randomly throughout the regions that are now the Kuiper belt.
A simplistic hypothesis, then, is that the expected chemical gradients in the primordial disk (Bergin et al. 2007) lead to gradients in the composition of the solid bodies. The large sold bodies, however, will move differentially through the disk while growing, incorporating materials from diverse regions (Weidenschilling 1997), so the clear chemical boundries of the disk are unlikely to be maintained in the macroscopic bodies. Indeed, short period comets, derived from the Kuiper belt, show strong evidence for a chemical diversity (i.e. Bockelée-Morvan et al. 2004; Dello Russo et al. 2009). which is unexplainable as a simple difference in formation location

In light of the large variability of cometary nuclei, we seek a hypothesis for the colors of the KBOs in which sharp color divisions can occur even in the presence of substantial chemical variability. We consider the possibility of a surface evaporation gradient in the disk. In our scenario KBOs form out of a variable mix of cometary materials and, as they are exposed to sunlight when the dust and gas disk first disappear, their surfaces evolve. Depending on the heliocentric distance dependent thermal equilibrium temperature, different molecules will evaporate and be lost to space. Surfaces of objects further from the sun will retain more volatile molecules that are lost on the surfaces of objects closer to the sun. When a Nice-like instability occurs and the objects are scattered further from the sun, their temperatures drop and evaporation of the less volatile species ceases. Long term irradiation develops the colors currently seen on the surfaces through chemical modification of whatever remains.

To quantitatively explore this scenario we use the volatile loss model of Schaller \& Brown (2007) and examine evaporation as a function of object size and heliocentric distance. We calculate the minimum loss of molecular species by assuming Jeans escape - the slowest possible escape mechanism - throughout the primordial disk. We calculate the size-dependent heliocentric distance at which all of a particular volatile should be depleted (Fig. 1).

Because of the exponential nature of Jeans escape, most specific model parameters chosen have little impact on the final result, but for concreteness we describe them here. We chose all parameters to be within the range of plausible values. Objects are assumed to have densities of $1.5 \mathrm{~g} \mathrm{~cm}^{-3}$, albedos are chosen to represent initially frosty objects and are identical to Pluto, starting compositions are those measured in the Hale-Bopp coma (Bockelée-Morvan et al.2004), with inert rock to give the correct final density. Even order-of-magnitude variations in the abundances do not change the conclusions below. We consider all species with measured abundances relative to water of $0.5 \%$ and higher, with the exception of $\mathrm{H}_{2} \mathrm{CO}$, for which no accurate laboratory data on the vapor pressure over the solid exists. All other vapor pressure are taken from the compilation of Fray \& Schmitt (2009). The sun during this early stage is assume to have $70 \%$ of the current energy output (Gough 1981).

Two model parameters can affect the final result substantially. First, we can either assume that the entire object is porous and that volatiles throughout the body have access to the surface and are capable of eventual evaporation, or we can assume that only a layer near the surface can evaporate. The final colors of Kuiper belt 
objects depend only on the chemistry in a very small layer near the surface, so both types of objects would appear identical on the surface, but the amount of material that needs to be evaporated for an object to appear depleted differs greatly in these two scenarios. Even very small short period comets still appear to have volatiles such as CO, which would quickly evaporate in the surface layers of almost any object in the primordial Kuiper belt (Bockelée-Morvan et al.|2004). We thus assume that evaporation is only a surficial process and we set the depth required to deplete the surface to $100 \mathrm{~m}$. Changing this depth by orders of magnitude in either direction does not qualitatively change the conclusions below.

The second important assumption is the length of time that passes from when the disk dissipates and the objects are exposed to sunlight to when the objects are scattered to their more distant locations. In the canonical Nice model, the scattering by the planetary instability is the cause of the Late Heavy Bombardment 650 Myr after the formation of the solar system (Gomes et al. 2005), so the primordial objects spend a long period of time closer to the sun. Such a long period is not a requirement of an instability model, however, so the actual time remains unconstrained. Regardless of the exposure time, an irradiated crust can develop much more quickly (Hudson et al. 2008), so additional exposure time can no longer affect colors. We set our exposure time to be 10 Myr, but, again, values differing by an order of magnitude in either direction do not qualitatively change the conclusions below.

For a wide range of assumptions, evaporation in the early Kuiper belt behaves as shown in Figure 1. Water ice is involatile at all distances and $\mathrm{CO}_{2}$ and $\mathrm{H}_{2} \mathrm{~S}$ are involatile throughout the Kuiper belt. Objects residing in the outer parts of the Kuiper belt retain $\mathrm{CH}_{3} \mathrm{OH}$, then $\mathrm{C}_{2} \mathrm{H}_{2}, \mathrm{C}_{2} \mathrm{H}_{6}$, and $\mathrm{HCN}$ over a small range of distances. $\mathrm{NH}_{3}$ is retained only near the distance of the current cold classical Kuiper belt, and $\mathrm{CH}_{4}, \mathrm{~N}_{2}$, and $\mathrm{CO}$ are depleted on surface layers throughout the Kuiper belt except for the largest objects. For the specific parameters chosen, the evaporation line of $\mathrm{CH}_{3} \mathrm{OH}$ appears near 20 $\mathrm{AU}$, which would be in the middle of the primordial disk of KBOs.

\section{SURFACE COLORS}

Experiments on ice irradiation in the outer solar system have primarily focused on specific chemical pathways and species rather than less precise coloration (Hudson et al. 2008), nonetheless some trends appear clear.

Objects that form between the inner edge of the primordial Kuiper belt and approximately 20 AU will have temperatures sufficiently high that the only major ices that remain on the surface are $\mathrm{H}_{2} \mathrm{O}, \mathrm{CO}_{2}$, and (with a much smaller abundance) $\mathrm{H}_{2} \mathrm{~S}$. No specific experiments have been done on the coloration or albedo of such a mixture after irradiation, but irradiation of $\mathrm{H}_{2} \mathrm{O}, \mathrm{CO}_{2}$ mixtures is known to produce carbonic acid and more complex hydrocarbons (Moore et al. 1991; Delitsky \& Lane 1998). Irradiation of such hydrocarbons then leads to the loss of hydrogen, the production of larger carbon chains, and the eventual carbonization of the surface. The final product is a dark neutrally colored spectrally bland surface (Andronico et al. 1987; Moroz et al. 2004;

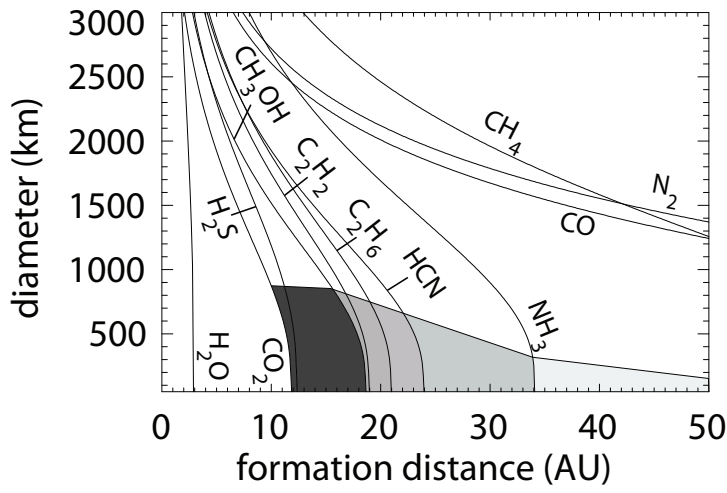

FIG. 1.- Evaporation gradients on surfaces in the early solar system. Objects to the right of the lines have that molecular species depleted from a $100 \mathrm{~m}$ layer in 10 Myr Changing the size of the depleted region or the depletion time scale by an order of magnitude only moves the locations of the curves by approximately an AU. The shaded regions show the formation regions of the three main surface types of Kuiper belt objects. In the inner part of the Kuiper belt, only $\mathrm{H}_{2} \mathrm{O}$ and $\mathrm{CO}_{2}$ survive on the surface. Irradiation of these molecules causes a dark neutral surface. In the middle part of the primordial Kuiper belt $\mathrm{CH}_{3} \mathrm{OH}$ is retained on the surface, leading to higher albedo redder surfaces. In the region of the current cold classical Kuiper belt, $\mathrm{NH}_{3}$ is retained, leading to the unique surface characteristics of these objects.

Palumbo et al. 2004). Irradiation of a $\mathrm{H}_{2} \mathrm{O}, \mathrm{CO}_{2}$ mix has been speculated to be a cause of the very dark crust of Callisto (McCord et al. 1998).

Such surfaces describe the dark neutrally colored centaurs well. In addition, with the exception of some of the larger water ice rich KBOs, the neutral KBOs have low albedos similar to those of the centaurs (Stansberry et al. 2006). We propose that the objects that are now the neutral-colored KBOs were formed in the inner part of the primordial disk and scattered into the current Kuiper belt. When they scatter inward to become centaurs their temperatures do not increase markedly beyond those that were experiences at formation, so surfaces do not evolve significantly.

Beyond approximately $20 \mathrm{AU}$, several major hydrocarbon species can remain on the surface. The most abundant of these is methanol. A Raman study of residues remaining after methanol irradiation showed that the methanol residue is surprisingly lacking in the signature of amorphous carbon (Ferini et al. 2004). Indeed, Brunetto et al. (2006) have shown that methanol, when irradiated to dosages expected for solar system aged KBOs (Cooper et al. 2003), does not turn dark and neutral but instead retains higher albedos and redder colors. The colors and albedos are similar to those seen in the red centaurs and likely also the medium-size red KBOS. Methanol, intriguingly, is also the only involatile molecule other than water identified either on a centaur (Cruikshank et al. 1998) or a KBO (Barucci et al. 2006). We propose that the presence of methanol on the primordial surface of a $\mathrm{KBO}$ allows that KBO to maintain a higher albedo redder irradiation crust. It is possible - indeed likely - that that the $\mathrm{C}_{2} \mathrm{H}_{6}$ and the $\mathrm{C}_{2} \mathrm{H}_{2}$ and $\mathrm{HCN}$ evaporation lines, which are just beyond the methanol line effect colors also. We thus expect the red objects to exhibit a wider range of colors than the more uniform neutral objects. These red objects, when scattered into the centaur region, also need not evolve significantly, as the red irradiation mantle is stable to higher temperature 
than pure methanol.

The neutral and red KBOs scatter throughout the outer solar system, and in the classical Nice model they are responsible for populating the cold classical Kuiper belt (Levison et al. 2008). Such a formation is inconsistent with the observation that the cold classical objects are composed purely of red objects and have other unique characteristics. Recently, however, Batygin et al. (2011) have shown that the cold classical belt need not have been emplaced during the Nice instability but rather could have survived if formed in place. Objects formed beyond $35 \mathrm{AU}$ would have been at all times beyond the ammonia evaporation line; No laboratory experiments have tested whether the addition of ammonia will have a significant effect on coloration after irradiation, but ammonia has been shown to block some types of irradiation chemistry (Hudson \& Moore 2001), which could possibly affect coloration. Ammonia is the only major species to have an evaporation line outside of the expected primordial Kuiper belt but inside of the current cold classical Kuiper belt. We thus propose that the addition of ammonia is the cause of the unique colors and high albedos of the cold classical KBOs.

In our scenario, little evolution occurs as KBOs move into the centaur region. The non-cold classical KBOs should, then, have an identical color distribution as the centaurs. We examine whether the updated ground based color database1 of Hainaut \& Delsanti (2002) supports this implication. We select all objects with high quality color measurements (color gradient errors under 10\%) and absolute magnitudes between $H=6$ and $H=9$ (where measurements exist of both KBOs and centaurs, so size-related effects are minimized). We further separate those into the 19 with perihelion inside of 20 AU, to represent centaurs which could have thermally evolved and all 83 non-cold classical objects with perihelion greater than $30 \mathrm{AU}$, to represent an unevolved population. Histograms of these color distributions are show in Figure 2. The distributions do not appear to be significantly different. Indeed, a K-S test shows that the two populations are distinguishable only at the $48 \%$ confidence level, or, to state the result clearly, there is no evidence from the ground-based data that the centaur and the non-cold classical KBO colors are drawn from different populations. While this result sounds surprising given the long history of comparison of colors of centaurs and KBOs (reviewed in Doressoundiram et al. 2008), this is the first test of this specific subpopulation. The fact that these two populations are currently indistinguishable does not mean, of course, that they are identical. Further color observations will be crucial to further test this prediction.

\section{DISCUSSION}

We propose that evaporation of volatiles and subsequent irradiation of the remaining ices are the basis for the diverse colors of the Kuiper belt. The existence of three major zones of volatile evaporation in the primordial Kuiper belt gives rise to three major types of surface compositions. Laboratory experiments, when available, support the correlation of the colors and albedo with the specific volatiles expected to be retained on the surface.

\footnotetext{
${ }^{1}$ Compiled at http://www.eso.org/ ohainaut/MBOSS
}

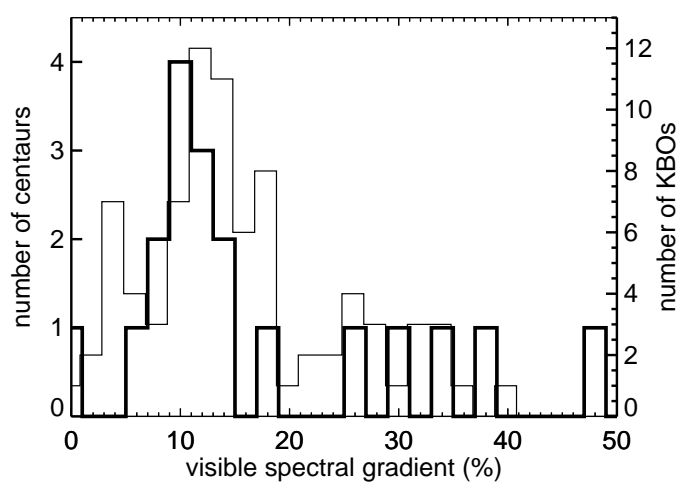

FIG. 2.- A comparison between the visible colors of centaurs (thick lines) and of similarly-sized non-cold classical KBOs (thin lines). A K-S test cannot distinguish any difference between these populations. While this statistical lack of difference is intriguing, more color data are needed - particularly of centaurs in this size range - in order to determine if the distributions are truly identical.

The hypothesis advanced here is necessarily nonunique. While the key physical process of evaporation of volatiles is inevitable, the interaction between the surface, the subsurface, the atmosphere, and space is sufficiently complex and unknown that more detailed modeling would give little additional insight. The few direct observational tests of this model test, at best, aspects only peripherally related, nonetheless, we predict: (1) Precision photometric observations will continue to show that the non-cold classical KBOs form two broad color groupings consistent with the centaurs of similar size; (2) With the exception of large KBOs and Haumea family members, all neutrally colored KBOs are similar in surface ice composition and will have the low albedos of the neutral centaurs; high precision albedo measurements may additionally show the small expected systematic albedo variation due to mixing with a non-ice component; (3) red KBOs will show slightly greater variability, but, with the exception of the largest objects, they will also not show a large range of albedos, but will have the elevated albedos of the red centaurs.

In lieu of powerful observational or theoretical tests, we expect that laboratory experiments will be the most useful for supporting or refuting this hypothesis. The studies used to suggest the chemical and color behavior here have been performed in a wide variety of laboratories concentrating on diverse subjects. For the hypothesis to be supported, a systematic laboratory study would have to show the following effects: (1) Water - carbon dioxide (and, possible $\mathrm{H}_{2} \mathrm{~S}$ ) ice mixtures must carbonize to form a neutrally-colored low albedo surface when irradiated at the appropriate level; (2) the addition of methanol to the ice mix must allow the irradiation product to remain red and have a higher albedo; and (3) the addition of ammonia to the mix must reproduce the unique colors and albedos of the cold classical Kuiper belt objects. While all of these possibilities are supported by the current laboratory data, only experimental verification will allow support or refutation of this hypothesis for explaining the colors of objects in the Kuiper belt.

This research has been supported by grant NNX09AB49G from the NASA Planetary Astron- 
omy program. We thank Hal Levison, Rosario Brunetto,

and Konstantin Batygin for enlightening conversations.

\section{REFERENCES}

Morbidelli, A. \& Brown, M. E. 2005, Comets II, ed. M.C. Festou, H.U. Keller, H.A. Weaver (U. Arizona Press: 2005)

Andronico, G., Baratta, G. A., Spinella, F., \& Strazzulla, G. 1987, A\&A, 184, 333

Barucci, M. A., Merlin, F., Dotto, E., Doressoundiram, A., \& de Bergh, C. 2006, A\&A, 455, 725

Batygin, K., Brown, M.E., \& Fraser, W.C., 2011, ApJ, in press

Benecchi, S. D., Noll, K. S., Grundy, W. M., Buie, M. W., Stephens, D. C., \& Levison, H. F. 2009, Icarus, 200, 292

Bergin, E. A., Aikawa, Y., Blake, G. A., \& van Dishoeck, E. F. 2007, Protostars and Planets V, 751

Bockelée-Morvan, D., Crovisier, J., Mumma, M. J., \& Weaver, H. A. The composition of cometary volatiles, ed. Festou, M. C., Keller, H. U., \& Weaver, H. A., 391-423

Brown, M. E. 2001, AJ, 121, 2804

Brown, M. E., Barkume, K. M., Ragozzine, D., \& Schaller, E. L. 2007, Nature, 446, 294

Brucker, M. J., Grundy, W. M., Stansberry, J. A., Spencer, J. R., Sheppard, S. S., Chiang, E. I., \& Buie, M. W. 2009, Icarus, 201,284

Brunetto, R., Barucci, M. A., Dotto, E., \& Strazzulla, G. 2006, ApJ, 644, 646

Cooper, J. F., Christian, E. R., Richardson, J. D., \& Wang, C. 2003, Earth Moon and Planets, 92, 261

Cruikshank, D. P., Roush, T. L., Bartholomew, M. J., Geballe, T. R., Pendleton, Y. J., White, S. M., Bell, J. F., Davies, J. K., Owen, T. C., de Bergh, C., Tholen, D. J., Bernstein, M. P., Brown, R. H., Tryka, K. A., \& Dalle Ore, C. M. 1998, Icarus, 135,389

Delitsky, M. L. \& Lane, A. L. 1998, J. Geophys. Res., 103, 31391

Dello Russo, N., Vervack, Jr., R. J., Weaver, H. A., Kawakita, H., Kobayashi, H., Biver, N., Bockelée-Morvan, D., \& Crovisier, J. 2009, ApJ, 703, 18

Doressoundiram, A., Boehnhardt, H., Tegler, S. C., \& Trujillo, C. Color Properties and Trends of the Transneptunian Objects, ed. Barucci, M. A., Boehnhardt, H., Cruikshank, D. P., Morbidelli, A., \& Dotson, R., 91-104

Ferini, G., Baratta, G. A., \& Palumbo, M. E. 2004, A\&A, 414, 757

Fraser, W. C., Brown, M. E., \& Schwamb, M. E. 2010, Icarus, 210,944

Fray, N. \& Schmitt, B. 2009, Planet. Space Sci., 57, 2053

Goldreich, P., Lithwick, Y., \& Sari, R. 2002, Nature, 420, 643
Gomes, R., Levison, H. F., Tsiganis, K., \& Morbidelli, A. 2005, Nature, 435, 466

Gough, D. O. 1981, Sol. Phys., 74, 21

Hainaut, O. R. \& Delsanti, A. C. 2002, A\&A, 389, 641

Hudson, R. L. \& Moore, M. H. 2001, J. Geophys. Res., 106, 33275

Hudson, R. L., Palumbo, M. E., Strazzulla, G., Moore, M. H., Cooper, J. F., \& Sturner, S. J. Laboratory Studies of the Chemistry of Transneptunian Object Surface Materials, ed.

Barucci, M. A., Boehnhardt, H., Cruikshank, D. P., Morbidelli, A., \& Dotson, R., 507-523

Jewitt, D. \& Luu, J. 1998, AJ, 115, 1667

Levison, H. F., Morbidelli, A., Vanlaerhoven, C., Gomes, R., \& Tsiganis, K. 2008, Icarus, 196, 258

Levison, H. F. \& Stern, S. A. 2001, AJ, 121, 1730

Luu, J. \& Jewitt, D. 1996, AJ, 112, 2310

McCord, T. B., Hansen, G. B., Clark, R. N., Martin, P. D.,

Hibbitts, C. A., Fanale, F. P., Granahan, J. C., Segura, M.,

Matson, D. L., Johnson, T. V., Carlson, R. W., Smythe, W. D., \& Danielson, G. E. 1998, J. Geophys. Res., 103, 8603

Moore, M. H., Khanna, R., \& Donn, B. 1991, J. Geophys. Res., 96, 17541

Moroz, L., Baratta, G., Strazzulla, G., Starukhina, L., Dotto, E., Barucci, M. A., Arnold, G., \& Distefano, E. 2004, Icarus, 170, 214

Nesvorný, D., Youdin, A. N., \& Richardson, D. C. 2010, AJ, 140, 785

Noll, K. S., Grundy, W. M., Stephens, D. C., Levison, H. F., \& Kern, S. D. 2008, Icarus, 194, 758

Palumbo, M. E., Ferini, G., \& Baratta, G. A. 2004, Advances in Space Research, 33, 49

Schaller, E. L. \& Brown, M. E. 2007, ApJ, 659, L61

Schaller, E. L., Brown, M. E., \& Haghighipour, N. 2009, AGU Fall Meeting Abstracts, B1213+

Stansberry, J. A., Grundy, W. M., Margot, J. L., Cruikshank, D. P., Emery, J. P., Rieke, G. H., \& Trilling, D. E. 2006, ApJ, 643,556

Stern, S. A. 2002, AJ, 124, 2297

Tegler, S. C., Bauer, J. M., Romanishin, W., \& Peixinho, N. Colors of Centaurs, ed. Barucci, M. A., Boehnhardt, H., Cruikshank, D. P., Morbidelli, A., \& Dotson, R., 105-114 Trujillo, C. A. \& Brown, M. E. 2002, ApJ, 566, L125

Tsiganis, K., Gomes, R., Morbidelli, A., \& Levison, H. F. 2005, Nature, 435, 459

Weidenschilling, S. J. 1997, Icarus, 127, 290 